\title{
AUTOMATED MODELING OF 3D BUILDING ROOFS USING IMAGE AND LIDAR DATA
}

\author{
N. Demir", E. Baltsavias \\ Institute of Geodesy and Photogrammetry, ETH Zurich, CH-8093, Zurich, Switzerland \\ (demir,manos)@geod.baug.ethz.ch
}

\section{Commission IV, WG IV/2}

KEY WORDS: Buildings, Multispectral classification, LiDAR data, DSM/DTM, Edge Matching, Detection, 3D Modelling

\begin{abstract}
:
In this work, an automated approach for 3D building roof modelling is presented. The method consists of two main parts, namely roof detection and 3D geometric modelling. For the detection, a combined approach of four methods achieved the best results, using slope-based DSM filtering as well as classification of multispectral images, elevation data and vertical LiDAR point density. In the evaluation, the combination of the four methods yields $94 \%$ correct detection at an omission error of $12 \%$. Roof modelling is done by plane detection with RANSAC, followed by geometric refinement and merging of neighbouring segments to clean up oversegmentation. Walls are then detected and excluded, and the roof shapes are vectorised with the alpha-shape method. The resulting polygons are refined using 3D straight edges reconstructed by automatic straight edge extraction and matching, as well as 3D corner points constructed by intersection of the 3D edges. The results are quantitatively assessed by comparing to ground truth manually extracted from high-quality images, using several metrics for both the correctness and completeness of the roof polygons and for their geometric accuracy. The median value of correctness of the roof polygons is calculated as $96 \%$, while the median value of completeness is $88 \%$.
\end{abstract}

\section{INTRODUCTION}

In this work, we focus on the 3D building roof modelling. Buildings have a critical role for 3D city models, decision support systems, governments, telecommunication, disaster management etc. Often, 3D building data are not available, not accurate enough, incomplete, or not up-to-date. Thus, methods are needed for generation of accurate and complete 3D building models with high degree of automation. Aerial images and LiDAR data are common sources for building extraction. In the past, some research dealt with building detection and reconstruction by using only image data (Henricsson and Baltsavias, 1997; Baillard et al., 1999; Brenner and Haala, 1998; Kada and McKinley, 2009), and some others by using LiDAR data (Maas and Vosselman, 1999; Rottensteiner, 2010; Elberink and Vosselman, 2009; Dorninger and Pfeifer, 2008). An updated review of building reconstruction methods can be found in Haala and Kada (2010). Image and LiDAR datasets have different strengths and weaknesses for object detection. LiDAR data provide $3 \mathrm{D}$ coordinates and generally no texture information. Image data have rich spectral information, but that turns into a disadvantage in urban areas because buildings have highly diverse spectral properties. On the other hand, LiDAR data can identify objects using geometric information. The capability of LiDAR data for object detection depends on the used filtering technique. LiDAR data miss some edge points, while image data has a clear advantage on edge features. In general, in order to overcome the limitations of image-based and LiDAR-based techniques, some researchers (Rottensteiner et al., 2005; Awrangjeb et al., 2010; Habib et al., 2010) combined these techniques. This is the main motivation for our work. Our methodology has two main parts, detection and 3D geometric modelling. This work is a significant extension of Demir and Baltsavias (2010).

\section{INPUT DATA}

The test site is in Vaihingen, Germany. The dataset has been provided from the DGPF camera evaluation project (Cramer,

*Corresponding author
2010). We have used DMC digital images with NIR, R, G, B channels and raw LiDAR point cloud. DMC images have $8 \mathrm{~cm}$ GSD. LiDAR points have a density of $5 \mathrm{pts} / \mathrm{m}^{2}$. There is no explicit verification of the accuracy of the LiDAR data, we assume the vertical positional error (sigma) as $20 \mathrm{~cm}$ with vertical differences $(18 \mathrm{~cm})$ caused by residual errors in the individual system components: GNSS, laser scanner and the mounting (Haala et al., 2010). In the text below, we use

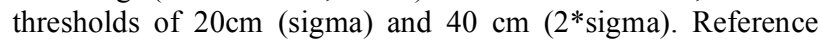
vector data has been collected by stereo measurement using the DMC images, with better than $15 \mathrm{~cm}$ accuracy. It consists of the planar roof polygons, which are larger than $25 \mathrm{~m}^{2}$ for 164 buildings.

\section{DSM \& DTM GENERATION}

Although LiDAR data is available in our test, we initially preferred to use an image-based DSM, which can always be generated densely with high-resolution images (though later we also used the LiDAR data due to reasons explained in section 4.4). For the DSM generation, the NIR channel is chosen because of its better contrast on edge features. The noise in the images is reduced using an edge-preserving filter. Then, a Wallis filter is applied to enhance the contrast. A DSM is generated with $25 \mathrm{~cm}$ grid spacing using the matching method by Zhang and Gruen (2004). A DTM is computed for extracting the above-ground objects. A slope-based progressive morphological filtering method (Zhang et al., 2003) has been used to reduce the DSM to DTM. An opening (erosion + dilation) is performed on the DSM to gain a secondary surface. The elevation difference of a grid between the previous and current surface is compared to a threshold to decide, if a grid point is a ground measurement. The height difference threshold has been computed using the predefined maximum terrain slope as defined by Zhang et al. (2003):

$d h_{T, k}=\left\{\begin{array}{ccc}d h_{\max } & \text { if } & d h_{T, k}>d h_{\max } \\ s\left(w_{k}-w_{k-1}\right) c+d h_{0} & \text { else if } & w_{k}>3 \\ d h_{0} & \text { else if } & w_{k} \leq 3\end{array}\right\}$

where $d h_{t, k}$ is the height difference threshold, $d h_{0}$ is the initial elevation difference threshold which approximates the error of 
DSM measurements (sigma of $0.2 \mathrm{~m}$ ), $d h_{\max }$ is the maximum elevation difference threshold $(3 \mathrm{~m}), s$ is the predefined slope $(0.10), c$ is the grid size and $w_{k}$ is the filtering window size (in number of cells) at the $k^{\text {th }}$ iteration. The size of filtering windows $w$ has been increased till the maximum building size was reached $(200 \mathrm{~m})$ and the derived surface has been used as an input for the next iteration. After generation of the DTM and subtracting it from the DSM, we get the normalised DSM (nDSM). Using the $\mathrm{nDSM}$ and a threshold of $1.5 \mathrm{~m}$, we get the above-ground objects (blobs). The above filtering approach was used to have full control of the code used due to project requirements, but there are commercial programs (e.g. SCOP++ LiDAR) that perform a better filtering.

\section{BUILDING DETECTION}

The buildings are detected with several methods, while each approach has different characteristics. A combination is proposed to achieve the best detection result. The approach starts with detection of above-ground objects in the DSM. Then, the first approach uses all possible spectral information to detect the buildings; the second one eliminates the trees from the detected above-ground objects not using all spectral information, but only NDVI. While urban areas have diverse spectral information, the buildings are detected in these two methods with maximum and minimum spectral information. The third approach detects the above-ground objects in the LiDAR data, and eliminates the trees using image data to detect only buildings. The fourth approach is fully based on the LiDAR data, so it does not include any drawbacks from image data, but has weaknesses, especially on building outlines, which are detected as trees. Fig. 1 shows the workflow for the building detection. The initial rationale for this procedure is to test and compare various simple known approaches (with the exception of method 4 which was developed by us) and then compare these results to their combination, which would be hopefully better. A posteriori we realised that: a) the DSM and DTM could be computed either from image matching (possibly using also a LiDAR DTM to derive the $\mathrm{nDSM}$ ) or only LiDAR data; and b) the multispectral classification could use an optimal combination of channels and not only NDVI as in method 2. In case only images or only LiDAR data are available, it is also possible to detect the buildings, albeit less accurately, using a subset of the methods below.

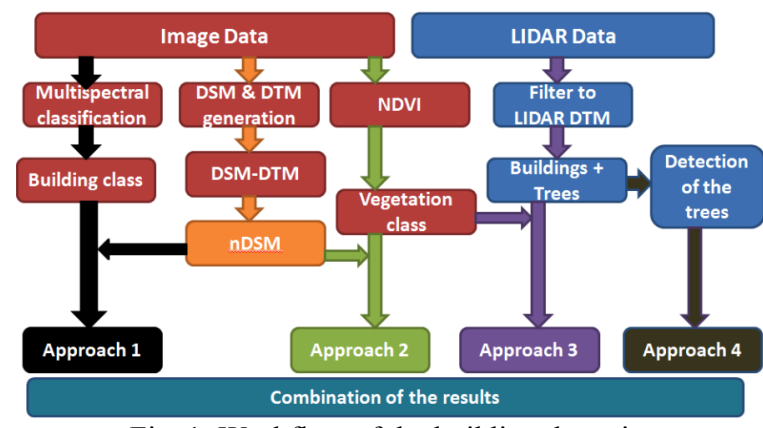

Fig. 1. Workflow of the building detection.

\subsection{Multispectral classification}

In this approach, a pixel-based supervised classification is used to detect buildings. The available channels are NIR (Near InfraRed), R (red), G (green), B (blue). The used classes are buildings, bare ground, roads, shadows, grass and trees. Principal components (PC), NDVI and saturation are added channels. NIR, R, PC1 ( $1^{\text {st }}$ PC), NDVI, saturation are selected using divergence analysis to do a maximum likelihood classification using ERDAS software. Using the height information from the nDSM, the mixed building and ground class could be separated. $94 \%$ of the building class is correctly classified, the omission error is $15 \%$. The omission error mostly comes from shadows and vegetation on the roofs and errors in DSM filtering.

\subsection{Using the blobs and NDVI classification}

ISODATA clustering of the NDVI image extracts the vegetation and no vegetation regions. Finally, the intersection of no vegetation regions and blobs extracts the buildings. The correctness of the buildings is calculated as $91 \%$ and the omission error is $15 \%$. Errors in filtering and vegetation on the roofs negatively affect the completeness.

\subsection{Filtering of LiDAR point cloud and NDVI classification}

In this approach, the LiDAR DTM (non-interpolated) is used to detect the above-ground objects. The LiDAR DTM is derived from the raw LiDAR point cloud by using the SCOP++ LiDAR package. The buildings are voids and the trees have low density $\left(<2\right.$ points $\left./ \mathrm{m}^{2}\right)$ in the LiDAR DTM, thus buildings and trees are estimated. Using the vegetation class from the NDVI classification as a mask, removes trees, while the remaining objects (buildings) are deleted, if their area is smaller than $25 \mathrm{~m}^{2}$. The correctness of the buildings is $81 \%$ and the omission error is $12 \%$. The omission error mostly comes from removing vegetated roofs. The error in filtering affects the completeness.

\subsection{Detection of the trees from raw LiDAR point cloud}

The fourth method is based on the raw LiDAR point cloud. The detection of the trees is not always convenient when using image data, because roof surfaces may be covered by trees or may contain a green surface. Then, these surfaces are detected as vegetation using any image classification process. Usage of the LiDAR data avoids this kind of problems since the detection is based on the geometry of the objects. In the LiDAR data, the vertical point density is generally much higher at trees than at open terrain or buildings. We start from the above-ground objects, which were detected with the LiDAR data filtering of section 4.3. Then, the RANSAC method (Schnabel et al., 2007) is applied on all above-ground points to find planar surfaces, which belong to the buildings. The details of finding planar surfaces will be discussed in section 5.1. After the removal of all planar objects, all the remaining points consist of the building roofs, which do not have planar surfaces, other objects e.g. cars and the tree regions. The process is followed by a search window over the LiDAR data with a size of $5 \mathrm{~m} \times 5 \mathrm{~m}$. The size of a search window is selected with consideration of the minimum tree size. Secondly, the search windows are subdivided into eight equal regions in vertical space. The number of sub-regions $v d$ which contain points are counted to calculate the vertical density in the search window. The point density $d$ of the search window and the tree height $z$ are calculated as well. The parameters are estimated by empirical tests, so $v d$ is chosen as bigger than $50 \%, z$ as $3 \mathrm{~m}$ and $d$ as 15 $\mathrm{pts} / 25 \mathrm{~m}^{2}$ for our tests. The buildings are derived by removing tree points from the above-ground objects. The correctness of the buildings is $80 \%$ and the omission error is $20 \%$. The walls of the buildings are extracted as trees because of their high vertical density due to laser pulse double-bounce or multiple echoes. This affects the quality of the completeness and the correctness. 


\subsection{Combination of the detection results}

Binary operations are used for the combination of the results. The combination of the results is performed based on consideration of the properties of the used datasets and the advantages and disadvantages of each method. The results from the image data depend on the spectral information. Vegetation on roof surfaces is one of the existing problems. Secondly, the shadows on the buildings do not belong to the building class, and this negatively affects the completeness. Additionally, detected shadows on vegetation are detected as buildings in the second approach, which uses NDVI classification. Intersection of the results from the image data minimizes correctness errors, but it affects the completeness negatively. Furthermore, LiDAR-based results are used in the union process since they are not affected by the spectral problems mentioned above. Additionally, the final detection result is supposed to be included in the above-ground objects. Thus, finally, an intersection is performed with the detected blobs. So, the suggested binary operation is (R1 $\cap \mathrm{R} 2 \mathrm{U}(\mathrm{R} 3 \mathrm{U}$ R4) $) \cap$ Blobs. $\mathrm{R}$ represents the results from the four approaches. In the evaluation, the combination of these steps yields $94 \%$ correct detection at an omission error of $12 \%$. The final result is shown in Fig. 2. The binary operations were used because all partial results were also binary. A better approach would be to derive probabilities for each detection method that each pixel is a building, and then combine the results using a weighted scheme.

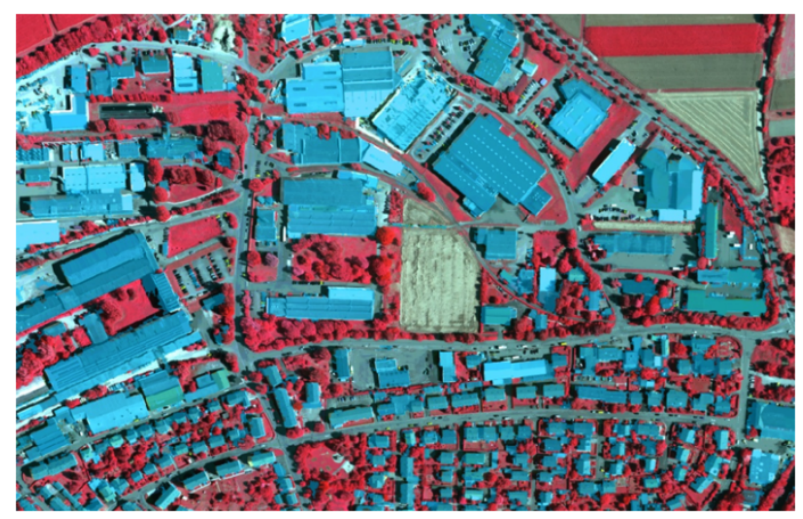

Fig. 2. Final building detection result (blue: buildings).

\section{ROOF RECONSTRUCTION}

The workflow of roof reconstruction is shown in Fig. 3. The approach starts with detection of planes and finalizes the reconstruction with regularization of the roofs.

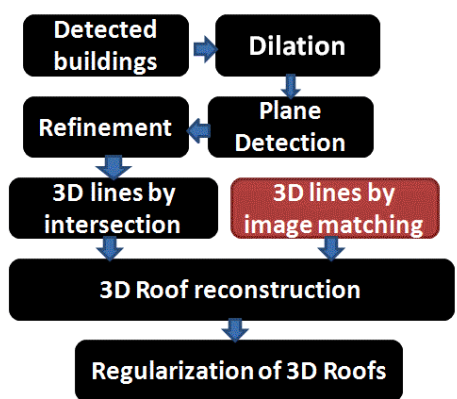

Fig. 3. Workflow of the reconstruction.

\subsection{Detection of roof planes}

First, dilation is applied on the detected buildings to include most LiDAR points, which belong to buildings. The dilation threshold is selected as $2 \mathrm{~m}$ empirically. Then, the surface normal vectors are calculated. It is supposed that each point, which belongs to a planar surface, should have a similar surface normal vector with its neighbours. The minimum size of the detectable roof features is the main decisive fact to choose the number of the neighbour points. Multiplying the size of the minimum detectable feature $s\left(\mathrm{~m}^{2}\right)$ with the density of the point cloud per $\mathrm{m}^{2} d$ provides the number of the neighbour points $P$. In our case, $s$ is selected as $4 \mathrm{~m}^{2}$ for considering the minimum possible roof plane area, $d$ is $5 \mathrm{pts} / \mathrm{m}^{2}$, so $P$ is calculated as 20 points. The plane coefficients $a, b, c$ are calculated by least squares fitting, calculated coefficients are associated with every point in the LiDAR data. Now, the input data for the plane detection are $\mathrm{x}, \mathrm{y}, \mathrm{z}$ coordinates and associated plane normal vectors of the points. The detection is based on the RANSAC approach (Schnabel et al., 2007). Using RANSAC, in each iteration, the planar surface with the maximum score is searched. Candidate shapes are generated for every minimal set of the points in a predefined distance threshold of $40 \mathrm{~cm}$. To confirm the created plane, the angle between surface normal vectors is determined. The angle $\gamma$ between two normal vectors is computed. The candidate plane is accepted only if this angle $(\gamma)$ is less than the predefined angle $\alpha\left(\right.$ chosen as $\left.10^{\circ}\right)$. The other parameter is the minimum number of the points, which belong to one planar surface, which was set to 20 points as explained above. Some examples from detected planes overlaid on an orthoimage are shown in Fig. 4.

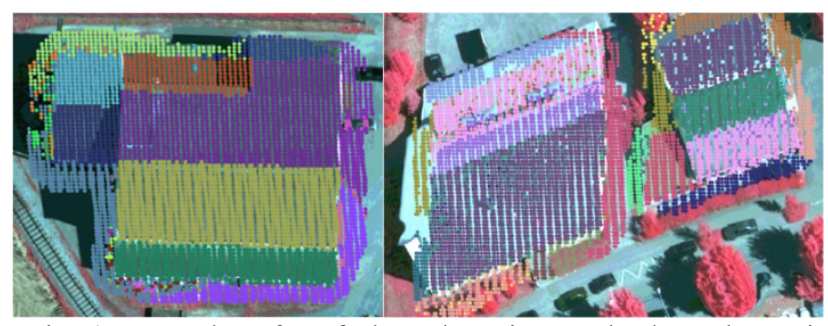

Fig. 4. Examples of roof plane detection, each plane shown in another colour.

\subsection{Refinement of the plane detection}

The refinement consists of two steps; assignment of the points to a better plane, and secondly, a region growing approach. Additionally, the planes are classified as roofs, walls and ground.

The process starts with calculation of the distance $D$ from each point to all detected planes in each building as follows:

$$
D=\left(a x_{0}+b y_{0}+c z_{0}\right) / \sqrt{a^{2}+b^{2}+c^{2}}
$$

where $a, b, c$ are the plane coefficients and $x_{0}, y_{0}, z_{0}$ are the coordinates of the point.

Let $P$ be any point on the building roof, the plane A is from the first assignment, any other plane on the building is B. The conditions for the refinement are defined as:

$$
\text { if }\left(N_{b}>N_{a} \&\left|D_{a}-D_{b}\right|<\sigma \text { and } \gamma<\alpha\right)
$$

where $D a$ is the distance between point $\mathrm{P}$ and $\mathrm{A}, D b$ is the distance between point $\mathrm{P}$ and $\mathrm{B}, \mathrm{Na}$ is the number of the points in $\mathrm{A}, N b$ is the number of the points in $\mathrm{B}, \sigma$ is vertical positional error of the LiDAR data.

According to Eq. (3), the plane assignment is changed from plane A to plane $B$, if the difference between the distances to assigned plane A and the plane B is smaller than the accuracy of the LiDAR data and the number of points in plane B is bigger 
than the number of the points in plane A. The angle between planes A and B is also calculated; this angle should be less than the predefined angle $\alpha$, which is chosen identical as in the plane detection step. To overcome the over-segmentation problem, a region growing approach is applied. Additionally, the slope and the height from the ground classify the planes whether they belong to the roofs, walls or the ground. The criteria are listed in Table 1. Fig. 5 shows the examples from the refined planes. Fig. 6 shows the plane classes with different colours. The quality assessment of the plane detection (Table 2) is discussed in section 7.

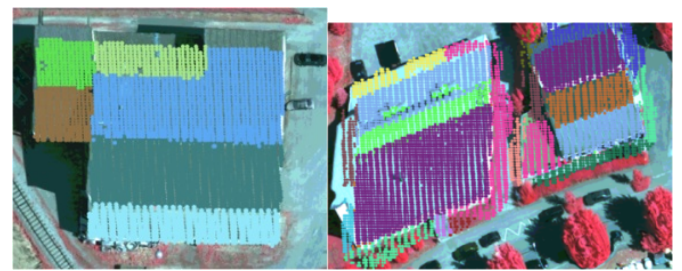

Fig. 5. Examples from the detected refined planes.

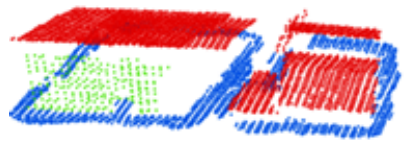

Fig. 6. Identified planes related to Fig. 5 right (red: roof, green: wall, blue: ground).

Table 1. Criteria for classifying the planes.

\begin{tabular}{|l|l|}
\hline Roof & Height $\geq 1.5 \mathrm{~m}$ slope $<85^{\circ}$ \\
\hline Wall & Height $\geq 1.5 \mathrm{~m}$ slope $\geq 85^{\circ}$ \\
\hline Ground & Height $<1.5 \mathrm{~m}$. \\
\hline
\end{tabular}

\subsection{Inner edge extraction}

The inner edges (Fig. 7) are generated by intersecting the neighbouring planes. To find the end points of the calculated line, a bounding box is created by using the points in the neighbourhood region of the planes. The closest points on the calculated edge to the maximum and minimum values of the bounding box are the end points of the intersection edges. The created edges are converted to dense points at every $40 \mathrm{~cm}$ for further processing.

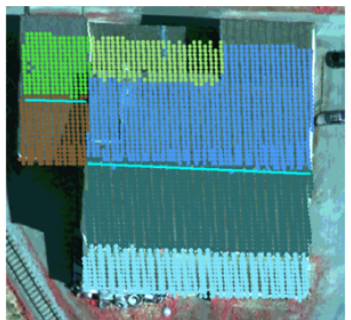

Fig. 7. Extracted inner edges (cyan).

\subsection{Alpha-shape algorithm for roof outlines}

The Alpha-shape algorithm (Edelsbrunner and Mücke, 1994) is used to reconstruct the roof shapes from the point set. The roof boundaries are extracted using the alpha-shape algorithm because it does not have any restriction for concavity like other boundary extraction methods, e.g. convex-hull. Firstly, initial outlines of the roof planes are extracted, and then they are refined with more geometrical features (3D edges from intersection and the images) and roof polygon regularisation.

\subsection{D straight edges from images}

3D edges are created by edge matching in the DMC images. They are integrated in the modelling process because image data has an advantage regarding modelling of surface discontinuities, especially building outlines, compared to LiDAR. Firstly, 2D edges are extracted with a two-stage approach: (i) extracting Canny edges, and (ii) robustly fitting them to the straight edges and performing matching using the method described in Ok et al. (2010). The straight edges which are not aligned with epipolar lines are found directly by intersecting the projection planes as proposed in Schmid and Zissermann (2000); the ones aligned with epipolar lines are reconstructed with the method proposed in Ok et al. (2010). The created straight edges are converted to points at every $40 \mathrm{~cm}$ for further processing. This parameter $(40 \mathrm{~cm})$ can be selected freely. The extracted 3D straight edges are shown in Fig. 8. These straight edges are used in section 6 .

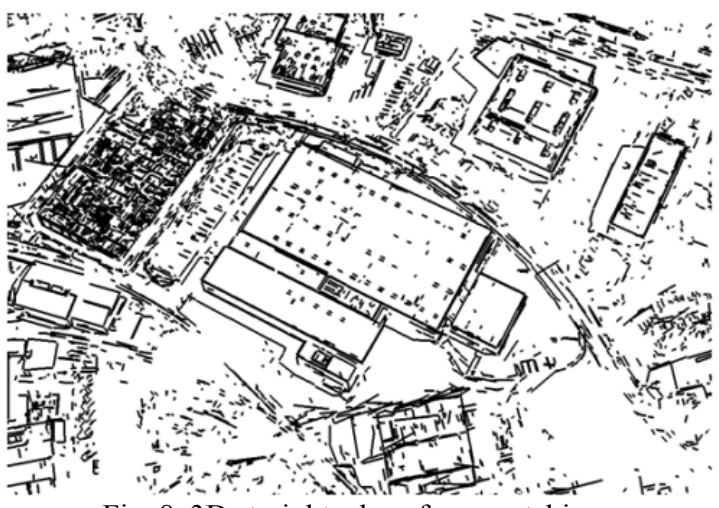

Fig. 8. 3D straight edges from matching.

\section{REFINEMENT OF ROOF OUTLINES}

\subsection{Assignment of 3D straight edges to planes}

The purpose of this step is using 3D straight edges from images where they exist, giving more precisely the roof outline. The problem is assignment of $3 \mathrm{D}$ straight edges to the corresponding roof planes. The assignment is performed for each $3 \mathrm{D}$ straight edge. Each 3D straight edge is assigned to the best corresponding planar surface as described below. Firstly, the 3D distance to the detected plane is calculated with:

$$
D=\left(a_{i} x_{0}+b_{i} y_{0}+c_{i} z_{0}\right) / \sqrt{a_{i}^{2}+b_{i}^{2}+c_{i}^{2}}
$$

where $a_{i}, b_{i}, c_{i}$ are the coefficients of plane $i, x_{0}, y_{0}, z_{0}$ are the coordinates of a point on the $3 \mathrm{D}$ straight line. And secondly, the closest Euclidian distance $d s$ between each edge point $\left(x_{1}, y_{1}, z_{1}\right)$ and point $\left(x_{2}, y_{2}, z_{2}\right)$ on the initial boundary of section 5.4 is calculated. After calculating $D$ and $d$ s, two criteria are investigated for assigning the points according to the detected planes. A point on the 3D straight edge is assigned to a plane, if $D$ is smaller than $20 \mathrm{~cm}$ and if $d s$ is smaller than $40 \mathrm{~cm}$. The threshold for $d s$ is chosen a bit larger to avoid problems occurring at the building outlines.

\subsection{Outline reconstruction}

The reconstruction process is performed by the alpha-shape algorithm for each planar surface separately. The points, which are associated with the detected planes, are used for the construction of the outline. The point set $P_{n}$ which is used in the 
reconstruction is calculated as: $P_{n}=P_{o d}+P_{\text {int }}+P_{3 \text { dline }}$ where $P_{n}$ shows the final points for reconstructing the roof surface, $P_{o}$ are the original LiDAR points with associated planes, $P_{\text {int }}$ are the converted points from the intersection edges, $P_{3 \text { dline }}$ are the assigned points of $3 \mathrm{D}$ straight edges from the images. Points in $P_{o}$ are eliminated in case they are close $(1 \mathrm{~m})$ to $\left(P_{\text {int }} \mathrm{U} P_{3 \text { dine }}\right)$. $P_{o d}$ is the new point set from $P_{o}$ after elimination. One example for the reconstructed roof outlines is in Fig. 9.

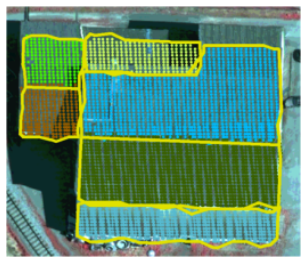

Fig. 9. Yellow lines: Reconstructed outlines of roof planes, other colours show the individual planes.

\subsection{Regularisation of the roof outlines}

The idea is finding the main straight edges of the roof outlines. Because the reconstructed outlines have irregular shapes where 3D straight edges from images are not available in section 6.1. For fitting the reconstructed outlines to the straight edges, a RANSAC approach is applied. The reconstructed outline is converted to dense points every $20 \mathrm{~cm}$. The created dense points are projected to $2 \mathrm{D}$ space. $\mathrm{z}$ values are calculated by the surface normal coefficients of the corresponding planes. After fitting $2 \mathrm{D}$ edges, they are intersected between each other, and the intersection points are kept if they are close to the initial reconstructed outline (Fig. 10). The distance threshold is selected as $2 \mathrm{~m}$, equal to the dilation threshold in section 5.1. In Eq. (5), the $x, y, z$ coordinates of intersection point $P$ of two edges $\mathrm{L}_{1}$ and $\mathrm{L}_{2}$ in $2 \mathrm{D}$ is calculated as:

$x=\frac{\left(x_{1} y_{2}-y_{1} x_{2}\right)\left(x_{3}-x_{4}\right)-\left(x_{1}-x_{2}\right)\left(x_{3} y_{4}-y_{3} x_{4}\right)}{\left(x_{1}-x_{2}\right)\left(y_{3}-y_{4}\right)-\left(y_{1}-y_{2}\right)\left(x_{3}-x_{4}\right)}$

$y=\frac{\left(x_{1} y_{2}-y_{1} x_{2}\right)\left(y_{3}-y_{4}\right)-\left(y_{1}-y_{2}\right)\left(x_{3} y_{4}-y_{3} x_{4}\right)}{\left(x_{1}-x_{2}\right)\left(y_{3}-y_{4}\right)-\left(y_{1}-y_{2}\right)\left(x_{3}-x_{4}\right)}$

$z=-\frac{d+a x+b y}{c}$

where edge $L_{1}$ is defined by two points $\left(x_{1}, y_{1}\right)$ and $\left(x_{2}, y_{2}\right)$, edge $L_{2}$ is defined by two points $\left(x_{3}, y_{3}\right)$ and $\left(x_{4}, y_{4}\right)$, and $a, b, c, d$ are the plane coefficients.

The intersection points are connected successively according to their order in the first roof reconstruction. The angle between two neighbour points is assumed to be at a right angle. If the point does not meet this constraint, it is not selected. The created edges are converted to points every $40 \mathrm{~cm}$. This parameter can be selected freely with consideration of the density of the LiDAR data. The point set $P_{n}$ for the final reconstruction is formulated as: $P_{n}=P_{o d}+P_{\text {reg }}$ where $P_{n}$ shows the final points for reconstructing the roof surface, $P_{\text {reg }}$ is the point set which has the created points from regularization, $P_{o}$ are the original LiDAR points with associated planes. The points in $P_{o}$ are eliminated in case they are close $(1 \mathrm{~m})$ to $P_{\text {reg. }}$. $P_{o d}$ is the new point set from $P_{o}$ after elimination. After this combination, points $P_{n}$ are used for the reconstruction by using the alpha-shape algorithm. All modelled roofs and some detailed examples are shown in Fig. 11.

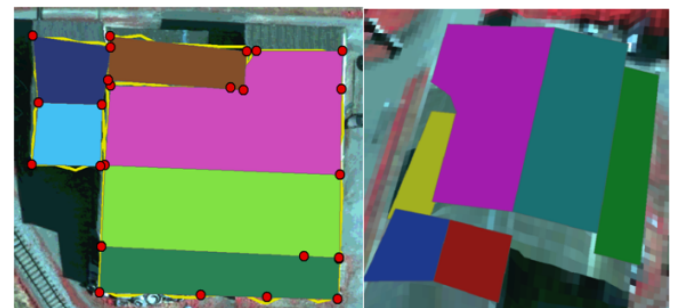

Fig. 10. Intersection points (red) of the roof outlines and reconstructed roof (Left: top view, right: 3D view).

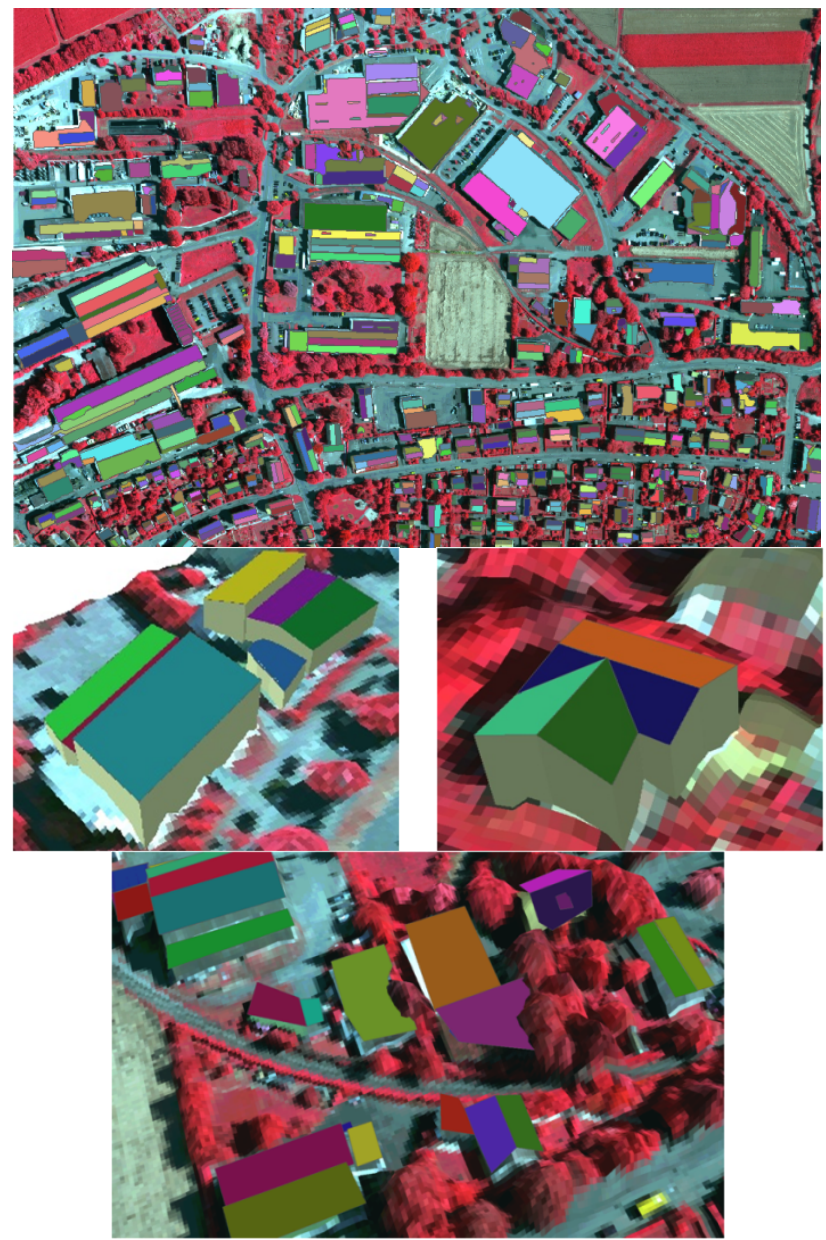

Fig. 11. All modelled roofs (top image) and some examples from modeled roofs with different colours for each individual roof surface.

\section{QUALITY ASSESSMENT}

Evaluation is performed for both approaches for plane detection and reconstruction. Four criteria are investigated for the plane detection (Table 2), while five criteria are investigated for the modelling (Table 3). If we denote the reference vector data as $\mathrm{R}$ and the roof model as $\mathrm{M}$, the reliability shows the ratio $(\mathrm{R} \cap$ $M) /(R U M)$. Correctness is calculated with $(R \cap M) / M$, while completeness with $(R \cap M) / R$. Average absolute distance between reference data and roof surface is calculated with $3 \mathrm{D}$ distances at every $20 \mathrm{~cm}$. Shifts in $\mathrm{X}, \mathrm{Y}$ and $\mathrm{Z}$ show the differences between building corner points in the reference data and the closest points on the outlines of the roof. 
Table 2. Accuracy assessment of the plane detection.

\begin{tabular}{|c|c|c|c|c|}
\hline & \multicolumn{2}{|c|}{$\begin{array}{c}\text { First detection } \\
\text { (Section 5.1) }\end{array}$} & \multicolumn{2}{c|}{$\begin{array}{c}\text { Refined detection } \\
\text { (Section 5.2) }\end{array}$} \\
\hline Criterion & Mean & Median & Mean & Median \\
\hline Reliability (\%) & 43 & 45 & 68 & 68 \\
\hline Correctness (\%) & 69 & 95 & 82 & 97 \\
\hline Completeness (\%) & 48 & 55 & 63 & 81 \\
\hline $\begin{array}{c}\text { Average absolute } \\
\text { distance (m) }\end{array}$ & 5.02 & 0.53 & 2.23 & 0.25 \\
\hline
\end{tabular}

From Table 2, it is clear that the refinement increases all quality measures.

Table 3. Accuracy assessment of the reconstructed roofs.

\begin{tabular}{|c|c|c|c|c|}
\hline & \multicolumn{2}{|c|}{$\begin{array}{c}\text { Reconstruction } \\
\text { without 3D } \\
\text { straight edges }\end{array}$} & \multicolumn{2}{|c|}{$\begin{array}{c}\text { Reconstruction } \\
\text { with 3D } \\
\text { straight edges }\end{array}$} \\
\hline Criterion & Mean & Median & Mean & Median \\
\hline Reliability (\%) & 71 & 76 & 65 & 79 \\
\hline Correctness (\%) & 83 & 97 & 84 & 96 \\
\hline Completeness (\%) & 78 & 85 & 76 & 88 \\
\hline $\begin{array}{c}\text { Average absolute } \\
\text { distance (m) }\end{array}$ & 0.64 & 0.23 & 0.54 & 0.14 \\
\hline Shift in X (m) & 0.16 & -0.06 & 0.06 & -0.02 \\
\hline Shift in Y (m) & -0.14 & 0.01 & -0.22 & -0.07 \\
\hline Shift in Z (m) & -0.03 & -0.10 & 0.15 & 0.08 \\
\hline
\end{tabular}

For each building, the quality criteria are calculated, and then the mean value is calculated by taking the average of all values for each criterion. The median is calculated by selecting the value in the middle after ordering of the values from low to high. Median values are more suitable to assess the results when outliers are present. From Table 3, it is evident that usage of 3D straight edges improves the quality of the modelled roofs.

\section{CONCLUSIONS}

In this study, we tried to detect and model building roofs using image and LiDAR data. Four different methods are presented to detect the buildings. Several items can be mentioned as pros of the proposed method. The buildings are detected and reconstructed with no prior information. The method is not model-driven, so the roof types are not restricted to any preexisting model library. Roof outlines are extracted based on the alpha-shape algorithm, there is no limitation on concavity of the roof planes. Combination of the methods provides the best correctness and completeness among the results. The spectral problems are eliminated using LiDAR data and height information, while the weakness of LiDAR data surface discontinuities is handled by using $3 \mathrm{D}$ edges from image matching. Some conclusions can be stated on limitations of the method. The roofs are assumed to be planar. Other types of the surfaces (cones, etc.) can be included in the RANSAC parameter estimation approach. The approach needs empirically defined thresholds, not ones based on model assumptions, a priori knowledge etc. However, the results mainly depend on quality and resolution of the input datasets. The roofs close to dense trees or vegetated roofs cause problems but can be partially modelled in case LiDAR data exist. 3D edges from images can be also used for inner edge reconstruction, while they can not be extracted in very small buildings in some cases. Walls are seldomly modelled (especially if no LiDAR data exist) and they are mostly generated by projecting the roof outline to a DTM. Regarding the DSM, DTM and nDSM, due to accuracy and economy reasons, either image matching (possibly in combination with a LiDAR DTM) or LiDAR data should be used. All cons could be subject of future research.

\section{ACKNOWLEDGEMENTS}

We acknowledge financial support from the projects EU FP6 Pegase, KTI Synergos and SSO DEMFuse as well as support regarding code and results by R. Schnabel and A.O. Ok.

\section{REFERENCES}

Awrangjeb, M., Ravanbakhsh, M., Fraser, C. S., 2010. Automatic detection of residential buildings using lidar data and multispectral imagery. ISPRS Journal of Photogrammetry and Remote Sensing 65 (5): 457-467.

Baillard, C., Schmid, C., Zisserman, A., Fitzgibbon, A., 1999. Automatic line matching and 3D reconstruction of buildings from multiple views. IAPRS ${ }^{*}$, Vol. 32, Part 3-2W5, pp. 69-80.

Brenner, C., Haala, N., 1998. Rapid acquisition of virtual reality city models from multiple data sources. IAPRS, Vol. 32, Part 5, pp. 323-330.

Cramer, M., 2010. The DGPF-Test on Digital Airborne Camera Evaluation

- Overview and Test Design. Photogrammetrie - Fernerkundung Geoinformation (2): 75-84.

Demir, N., Baltsavias., E., 2010. Combination of image and lidar data for building and tree extraction. IAPRS, Vol. 38, Part 3B, pp. 131-137.

Dorninger, P., Pfeifer, N., 2008. A comprehensive automated 3D approach for building extraction, reconstruction, and regularization from airborne laser scanning point clouds. Sensors 8(11): 7323-7343.

Edelsbrunner, H., Mücke. E. P., 1994. Three-dimensional alpha shapes. ACM Trans. Graph. 13(1): 43-72.

Elberink, S.O., Vosselman, G., 2009. Building reconstruction by target based graph matching on incomplete laser data: analysis and limitations. Sensors 9(8): 6101-6118.

Haala, N., Kada, M., 2010. An update on automatic 3D building reconstruction. ISPRS Journal of Photogrammetry and Remote Sensing 65 (6): $570-580$

Haala, N., Hastedt, H., Wolff, K., Ressl, C., Baltrusch, S., 2010. Digital Photogrammetric Camera Evaluation - Generation of Digital Elevation Models. Photogrammetrie - Fernerkundung - Geoinformation (2): 99 -115.

Habib, A.F., Zhai, R., Kim, C. 2010. Generation of complex polyhedral building models by integrating stereo-aerial imagery and LiDAR data. Photogrammetric Engineering \& Remote Sensing 76 (5): 609-623.

Henricsson, O., Baltsavias, E., 1997. 3-D building reconstruction with ARUBA: A qualitative and quantitative evaluation. In: Automatic Extraction of Man-Made Objects from Aerial and Space Images (II), Birkhauser, Basel, pp. 65-76.

Kada, M., McKinley, L., 2009. 3D building reconstruction from LiDAR based on a cell decomposition approach. IAPRS, Vol. 38, Part 3/W4, pp. 4752 .

Maas, H.-G., Vosselman, G., 1999. Two algorithms for extracting building models from raw laser altimetry data. ISPRS Journal of Photogrammetry \& Remote Sensing 54(2-3): 153-163.

Ok, A.O., Wegner, J.D., Heipke, C., Rottensteiner, F., Soergel, U., Toprak, V., 2010. A new straight line reconstruction methodology from multi-spectral stereo aerial images. IAPRS, Vol. 38, Part 3A, pp. 25-30.

Rottensteiner, F., 2010. Roof plane segmentation by combining multiple images and point clouds. IAPRS, Vol. 38, Part 3A, pp. 245-250.

Rottensteiner, F., Trinder, J., Clode, S., Kubik, K., 2005. Using the Dempster Shafer method for the fusion of LIDAR data and multi-spectral images for building detection. Information Fusion 6(4): 283-300.

Schmid, C., Zisserman, A., 2000. The geometry and matching of lines and curves over multiple views. Int. J. Comput. Vision 40(3): 199-233.

Schnabel, R., Wahl, R., Klein, R., 2007. Efficient RANSAC for PointCloud Shape Detection. Computer Graphics Forum 26 (2): 214-226.

Zhang, K., Chen, S., Whitman, D., Shyu, M., Yan, J.,Zhang, C., 2003. A progressive morphological filter for removing non-ground measurements from airborne LIDAR data. IEEE Transactions on Geoscience and Remote Sensing 41: 872-882.

Zhang, L., Gruen A., 2004. Automatic DSM Generation from Linear Array Imagery Data. IAPRS, Vol. 35, Part B3, pp. 128-133.

\footnotetext{
IAPRS: International Archives of The Photogrammetry, Remote Sensing and Spatial Information Science since Vol. 34; before International Archives of Photogrammetry and Remote Sensing.
} 